\title{
Role of Breastfeeding in Promoting Maternal \& Child Health and Policy Implications in New Zealand
}

\author{
Adhikari Ramil \\ Massey University, Wellington, New Zealand \\ Email: dr_ramils@yahoo.com
}

Received 2 August 2015; accepted 22 January 2016; published 25 January 2016

Copyright (C) 2016 by author and Scientific Research Publishing Inc.

This work is licensed under the Creative Commons Attribution International License (CC BY).

http://creativecommons.org/licenses/by/4.0/

(c) (7) Open Access

\begin{abstract}
Worldwide researches over infant's health have generally focused and aimed on understanding the complex factors (both positive and negative) affecting the infant's health which further had ultimately helped them to frame policies nationally as well as internationally. Out of these factors, breastfeeding is one of the vital concerns for research related to infant mortality and morbidity. The World Health Organization recommends that infants should be exclusively breastfed until first six months and receive nutritionally adequate and safe complementary foods thereafter while breastfeeding continues for up to two years of age or beyond. In New Zealand (NZ), only 12\% of 6 months old children were exclusively breastfed in 2006. The Ministry of Health plays a leading role for the protection, promotion and supporting breastfeeding in NZ. There are a number of areas/implications that NZ need to focus at such as providing antenatal and postpartum education, training of health professionals, community and workplace support, policy frameworks, breastfeeding statistics and intersectoral approach. These measures mainly focus at the initiation but less has been done to continue and support breastfeeding in NZ. In order to achieve this, all of these areas should be given prime and equal consideration. Hence, there is a strong need to develop strategies that maintain and promote breastfeeding at 6 months or beyond in NZ taking into considerations of various barriers and enabling factors.
\end{abstract}

\section{Keywords}

Breastfeeding, Benefits, Barriers, Policy Implications

\section{Introduction}

Worldwide researches over infant's health have generally focused and aimed on understanding the complex 
factors (both positive and negative) affecting the infant's health which further had ultimately helped them to frame policies nationally as well as internationally. Out of these factors, breastfeeding is one of the vital concerns for research related to infant mortality and morbidity [1]. Globally, the rate for exclusive breastfeeding up to 6 months of age was 20\% for Central and European countries as compared to $44 \%$ for South Asia. Breastfeeding (BF) is defined as the transfer of the human milk from the mother to the child that is the infant receives milk directly from mother's breast [2]. And Exclusive Breastfeeding (EBF) refers to that the infant receives no other form of milk or liquid for the first four months and that breast milk be used until one year of infant's age [3].Human milk is considered as tailor-made (well-suited) for the baby. It is believed to be the best start of infant's life because of its nutritional, immunological and psychological benefits to both mother and infant. Apart from the numerous health benefits of breast milk for mother and child, like in other developed countries, to promote breastfeeding as an optimum infant feeding practice has become an important and integral aspect of the New Zealand (NZ) public health agenda [4].In order to achieve this goal, it is very crucial to understand and increase awareness regarding the role of breastfeeding for both mother and child and also to minimize and control various barriers for the same. The objective of this research paper is to highlight the role of breastfeeding in promoting mother and child health followed by the discussion of policy implications in New Zealand.

\section{Background}

\subsection{International Breastfeeding Status}

No doubt, good nutrition in infancy promotes and strengthens optimum growth and development of the child. Infant feeding is not only providing nutrition to an infant but also is a psychological, social and educational interaction between parent and infant [2]. There had been significant evidence over the past decades that breast milk provides all the essential nutrients in well-balanced proportion thereby fostering growth and development of an infant which is impossible to mimic or replace with any other kind of food [5].

Globally, in the developing countries like Philippines, Zimbabwe and Nepal, overall 96.6\% (birth to 6 months)and $87.9 \%$ (6 to 12 months) of infants were currently breast-fed, $87.4 \%$ of 6 to 12 months old infants consumed water, $29.6 \%$ had other milk products, infant formula (15.1\%) and $41.0 \%$ of other liquids respectively [1]. In addition to this, the rate for EBF up to 6 months of age was $20 \%$ for Central and European countries as compared to $44 \%$ for South Asia [6]. In United Kingdom, according to Baby Friendly Initiative Statistics, $81 \%$ of babies were breastfed at birth. This figure got dramatically reduced to $69 \%$ by one week and $17 \%$ at 3 months, $12 \%$ and $1 \%$ at 4 and 6 months (EBF) respectively. The World Health Organization (WHO), American Academy of Pediatrics (AAP) and American Academy of Family Physicians recommends EBF for the first 6 months of infant's life and thereafter to start with nutritionally adequate and safe complementary foods along with the continuation of breastfeeding up to 2 years or more. Also, Healthy People 2010 (national health promotion and disease prevention initiative in the United States) aims to increase BF rates as $75 \%$ of all mothers to start BF at birth, 50\% of them to continue till 6 months, 25\% forEBF for 6 months and 25\% of mothers to continue breastfeed until 1 year of age [7].

\subsection{Breastfeeding Status in New Zealand}

Despite of these global targets, only 12\% of babies were EBF for 6 months in New Zealand. In the year 2005, it was found that the EBF rate on discharge into the baby friendly hospitals was about $80.5 \%$. This rate was $50 \%$ by the 6weeks and got further dropped by less than $40 \%$ till 3 months of age. In other words, the EBF rates were much higher at the time of birth or discharge from hospital but however there was a decline for the same up to 6 months thus highlighting a need for policy implications for promoting breastfeeding as a public health agenda and improving BF rates specifically at 6 weeks, 3and 6 months in New Zealand. Also, these BF rates had ethnic disparities and were very low among Maori, Pacific and Asian populations as compared to New Zealanders [8]. The current data from Plunket indicated that the EBF rates were higher but when they got combined with full BF rates, there was no much difference hence indicating this area as a highest need of concern. The full BF rates at 6 weeks and 3 months was $66 \%$ and 55\% respectively in the year 2006 [9].

\section{Benefits of Breastfeeding to}

\section{a) Infant}

Moving further, scientific evidence had acknowledged that BF is considered as a gold standard for the infant. 
It is believed to be the best start of infant life because of its nutritional, immunological and psychological benefits to both mother and infant. In terms of nutrition, human milk is considered as tailor-made (well-suited) for the baby. In other words, it provides the exact amount of correct nutrients in a well-balanced proportion at a right temperature as required by body of the infant [10].For instance, low amount of protein (Whey) and sodium content in the human milk prevents from overloading on the immature kidneys of the infant. It also provides essential minerals such as iron, zinc and calcium in order to meet the infant growth demands [11].

In addition to this, infant is born with immature immune system as well as the organs that may require a period of time to function at optimum level. Breast milk contains prebiotics (substances that enhance the growth of beneficial microflora), free fatty acids (FFA), mono glycerides, antimicrobial peptides, human milk glycans, bifidus factor, lysozyme, lacto peroxidase, lacto ferrin, lipoprotein lipase and epidermal growth factors (stimulate the gastrointestinal epithelium as a barrier) that are all responsible for developing and enhancing infant's innate immune system [12]. The presence of these antimicrobial, anti-inflammatory and immunologic stimulating agents protect the baby from diarrhea and other various infectious diseases such as respiratory and urinary tract infections, otitis media, necrotizing enterocolitis, bacterial meningitis and bacteremia [13]-[15]. Not only in the development of the immune system but the presence of essential and saturated fatty acids, medium chain triglycerides and long chain polyunsaturated fatty acids helps in the development of central nervous system (CNS) of the infant [11]. Overall breast milk is considered as the baby's first immunization. Breast milk gets more easily digested by the infant as compared to formula or any other milk and colostrum produced during the first 4 days of pregnancy helps in smoothing the passage of meconium (sticky dark stool in the bowels of baby during pregnancy) through the intestine [16].

The mechanic of sucking the breast milk directly from mother's breast helps in the development of jaw muscles and oral cavity. Sucking along with proper positioning of the infant during breastfeed promotes better jaw alignment and shaping of the hard palate that further results in straight and brace free smile. In addition to this, appropriate development of craniofacial structures helps in effective speech and communication patterns for the child in his/her later life [10]. Moving ahead, sudden infant death syndrome (SIDS) is drastically prevalent in New Zealand especially among Maori. During the period 2003-2007, 61.6\% of infants who died from SIDS were Maori. Now, though the research for the causes and prevention of SIDS is ongoing but BF with benefit of proper jaw and oral cavity formation can reduce the risk and incidence of SIDS [17]. EBF for the first six months of life protect the infant from various food allergies and asthma and also provides time for the infant's immune system to develop. Frequency and duration of breastfeeding matters a lot. The more and for longer duration the baby gets fed, lesser are the chances of getting infections and allergies [14].

Apart from the above mention benefits, breastfeeding may have several other minor benefits too. Breastfeed babies have fewer rashes from the diapers or nappies because of the low $\mathrm{pH}$ of bowels. It may also prevent picky eater syndrome that may occurs later in life as babies who are breastfeed for longer periods accept different taste more easily than who are not. Last but not the least, breastfeeding the infant, reduces the chances of lymphoma, leukemia and obesity which further prevents the risk of developing complications in later life such as heart diseases, stroke, high blood pressure, diabetes and many more and also have a positive effect on cognitive development and skills [18]. In other words, studies had shown that children who were ever breastfeed had more intelligence quotients (IQ) as compared to those who did not received [10].

\section{b) Mother}

Moving ahead, the considerable beneficial effects of breastfeeding on the health of the mother had been paid less attention in the past. Probably, this could be one of the reasons that had affected the overall breastfeeding rates too in the olden times. Currently number of researches have been conducted for the same and had proven plentiful amounts of beneficial effects for the mother in terms of physical, general health perception, mental health scores and mother's parity. Breastfeeding women are able to produce sufficient amount of milk even if the calorie intake is low. This increase caloric demand with breastfeeding helps the mother to be nutritionally sound and return to its pre-pregnant state far more quickly than those mothers who do not breastfeed. In addition to this, breastfeeding releases oxytocin into the body that helps in the uterine contractions and shrinking of the uterus. Also, breastfeeding women experience greater weight loss and fat reduction ultimately helping them to return to the pre-pregnant state [19]. Exclusive breastfeeding suppresses the ovulation that further delays mensuration for about 4 - 6 months of post partum period thus providing spacing and acting as a natural method for family planning. This method is known as Lactation Amenorrhea Method, LAM [7].

Not only this, there is significant evidence that breastfeeding reduces the risk of breast and ovarian cancers in 
the mothers [20]. Studies have proven that there is $4.3 \%$ reduction in the risk of developing breast cancer for each year a woman breastfeeds in her lifetime. In other words, women who had developed breast cancer, the average lifetime of breastfeeding duration in them is much shorter as compared to those in which the disease is absent [21]. One of the case control study of Sri Lankan women showed that increased duration of breastfeeding dramatically reduces the risk of developing breast cancer and there was a dose-response relationship between the two [22]. With regard to breastfeeding and ovarian cancer, agency of healthcare research and quality (AHRQ) performed a meta-analysis on 9 studies and found a reduction in the risk of ovarian cancer by $21 \%$ in breastfeeding women as compared to those who had never breastfeed [7].

Moreover, the increased time duration of lifetime breastfeeding has also been found to be associated with low risk of developing diabetes (Type 2) and hip fractures in postmenopausal women. Studies have also indicated that women who do not breastfeed are at higher risk of developing post partum depression (the cause for which is likely unclear), hypertension, hyperlipidemia and cardiovascular diseases. In other terms, breastfeeding has a positive impact on systolic and diastolic blood pressure of the mothers [23].

Apart from the physiological benefits, breastfeeding provides psychological support to both mothers and babies. Oxytocin is a hormone that passes form the mother to the baby through the breast milk. Both oxytocin and prolactin, known as "mothering" hormones plays a vital role in providing the sense of completion, satisfaction, well being, relaxation and mothering. Furthermore, going through a positive and supportive breastfeeding experience further helps the mothers to gain confidence, self-esteem and ego [19]. In a quasi-experimental study of Australian primipara's views about breastfeeding, mothers reported that after 6 weeks of time they feel like they "got into right" and had a wonderful and pleasurable feeling of increased determination of breastfeeding their child and described breastfeeding as a magical experience overall. Not only this, mothers identified the advantages of breastfeeding for their infant, for instance, enhancing their attachment, providing comfort, giving them something they loved, increasing their immunity and contributing to their babies blooming [24].

In another study to explore the maternal perceptions of early breastfeeding experiences and breastfeeding outcomes at 6 weeks, it concluded for the same. Mothers had explained positive feelings regarding breastfeeding experiences that included feelings of enhanced attachment, enjoyment of breastfeeding and a sense of pride and personal fulfillment [25]. Therefore, breastfeeding the baby not only helps the mothers physically but also provides a greater sense of psychological, intimate and emotional bonding with the infant. Breastfeeding nurtures both the mother and infant [26]. Even the baby feels warmth, identifies mother and her love which in turn in the later stages teaches him the lesson of love, sacrifice, friendship and relations. This has been proved in one of the study that aims to clarify or explore the role of breastfeeding in the mother-infant relationship. The results of the study concluded that a positive correlation exists between breastfeeding and mother-infant bonding [27].

Now, surprisingly breastfeeding has some societal and economic benefits, too. It is not only good for the mother and baby but is also beneficial for the environment and mother earth. One study showed that if each baby is bottle-fed, in the US, it might produce more than 86,000 tons of waste into the landforms in the form of cans and packaging [10]. Breastfeeding is cheap, money and time saving. It saves the costs of purchasing formulae milk and bottles. The US department of agriculture, published in 2001, estimated that a minimum of 3.6 billion could be saved if the breastfeeding rates met the goals of Healthy People 2010 [19].

\section{Breastfeeding Barriers to Mothers in NZ}

One of the main breastfeeding barrier for mothers is their early return to work. In NZ, The Parental Leave and Employment Protection Act of 1987 entitles 14 weeks of paid parental leave to the mother or either of the parent in a joint adoption in order to promote and improve breastfeeding rates in NZ [8]. The Department of Labor (DOL) had conducted a survey on the effect of paid parental leave and breastfeeding rates with the results of $84 \%$ of participants believed that paid parental leave does play a significant role however $20 \%$ of them thought that 14 weeks were insufficient to improve breastfeeding rates. This directly suggests that the short duration of paid parental leave is one of the major factor that contribute to many mothers returning to work much earlier than they would prefer ultimately having dropped breastfeeding rates at 3 and 6 months in NZ [28].

Societal norms and cultural beliefs also create hindrance for mothers to breastfeed. The Ministry of Health, 2003 maternity service consumer satisfaction survey found that $89 \%$ of women intend to breastfeed their babies and $94 \%$ of women breastfeed their babies either exclusively, fully or partially. In spite of this positive attitude, New Zealand women reported their concern with regard to lack of support from their partner/family members to 
continue breastfeeding. In addition, various cultural practices, such as cigarette smoking, obesity, sense of guilt, use of bottle-feeding and availability of infant formulae milk, low socio economic status especially with regard to Maori and Pacific communities all adds into the barriers list of breastfeeding in NZ [29].

\section{Policy Implications in NZ}

No doubt, the discussion above has provided a clear evidence of the beneficial role of breastfeeding in promoting maternal and child health and also regarding the dropped breastfeeding rates globally as well as in NZ. Now, with regard to policy implications in NZ for the same, the Ministry of Health (MOH) provides the leadership for breastfeeding strategies and policies and thus has set the following breastfeeding targets for NZ. Firstly, to increase the exclusive and full breastfeeding prevalence rate at 6 weeks to $74 \%$ by 2005 and $90 \%$ by 2010. Secondly, to increase the exclusive and full breastfeeding prevalence rate at 3 months to $57 \%$ by 2005 and $70 \%$ by 2010 and thirdly, to increase the exclusive and full breastfeeding prevalence rate at 6 months to $21 \%$ by 2005 and $27 \%$ by 2010 [30].

In order to achieve these targets, a number of interventions had been taken at the national, regional and local levels within NZ that can further be explained under three broad headings, that is practices or implications to protect, promote and support breastfeeding. First of all, under the protection, there are 3 main legislative framework and policies that have been formed and timely reviewed in order to protect breastfeeding in NZ. The Human Right Act 1993 protects the right to breastfeed by stating that a women has the right to breastfeed and the child to be breastfed, wherever mother and her child is otherwise permitted to and is protected from discrimination for breastfeeding. The Parental Leave and Employment Protection Act of 1987 is an other legislative measure that entitles 14 weeks of paid parental leave to the mother or either of the parent in a joint adoption in order to promote and improve breastfeeding rates in NZ [8]. Along with this, other employment or work related measures such as allowing breastfeeding breaks for mother who wish to breastfeed the infant at work, flexible working hours, onsite crèches and provision of appropriate facilities for expressing and storing breast milk were also included under the Employment Relations Act 2007. Another framework is Corrections Regulations 2005 that protect the right to breastfeed for the imprisoned mothers and to promote mother infant bonding by providing them the opportunity to stay with their babies for a minimum of six months and beyond up to 2 years [29].

Internationally and nationally, studies have indicated that there is a positive relationship between paid maternity leave and breastfeeding rates. In NZ, the Department of Labor (DOL) had conducted a survey on the effect of paid parental leave and breastfeeding rates with the results of $84 \%$ of participants believed that paid parental leave does play a significant role however $20 \%$ of them thought that 14 weeks were insufficient to improve breastfeeding rates. This directly suggests that the short duration of paid parental leave is one of the major factor that contribute to many mothers returning to work much earlier than they would prefer ultimately having dropped breastfeeding rates at 3 and 6 months in NZ [28]. Recently, there have been proposals made in the parliament of NZ in the year 2014 to increase paid parental leave from 14 to 26 weeks. This will definitely have a huge impact as of dose-response relationship and will provide a new direction towards the success and meeting $\mathrm{MOH}$ breastfeeding targets for NZ.

Moving further, there is a seven-point action plan which includes establishing a national inter-sectoral breastfeeding committee, implementing Baby Friendly Hospitals throughout New Zealand, gaining active participation of Maori and Pacific whanau, establishing consistent breastfeeding reporting and statistics, increasing breastfeeding promotion, advocacy and coordination, ensuring that pregnant women can access prenatal education and ensuring high quality and ongoing postpartum care for improving the initiation, promotion and maintenance of breastfeeding in New Zealand [8]. In other words, providing antenatal and postpartum education, training of health professionals, community and workplace support, policy frameworks, breastfeeding statistics and intersectoral approach are the main key areas or interventions that NZ should focus for promoting breastfeeding. All of these areas should be given prime and equal consideration for achieving MOH breastfeeding targets.

Breastfeeding is a learned experience. Hence, educating the mothers and family members play a vital role in the promotion and support for breastfeeding. Providing antenatal education to the mothers will not only helps with their decision to breastfeed but also prepare them mentally to start breastfeeding soon after the birth which is also widely supported by the baby friendly initiative into the hospitals [31]. This clinical practice of breastfeeding soon after birth is a strong support for the initiation of breastfeeding and as a result the breastfeeding 
rates are highest at birth within NZ. However, these interventions concentrate much on the initiation and less has been done to maintain the exclusive continuation of it up to 6 months or beyond [9]. Thus, supporting breastfeeding is very important within NZ so as to meet $\mathrm{MOH}$ breastfeeding targets.

Now, in context to supporting BF, various interventions like conducting workshops, seminars, public awareness campaigns must be addressed especially with regard to Maori and Pacific populations due to prevalence of lower breastfeeding rates among them. At present, there are two programs for improving breastfeeding rates in Maori known as B4Baby in South Auckland and Mum4Mum (peer support program) in the Hutt Valley [8]. There are other breastfeeding support organizations such as lead maternity cares (LMC), general practitioner services, Royal NZ Plunket Society, New Zealand Breastfeeding Authority (NZBA), Well Child services and other community health workers that aims to support and promote breastfeeding in NZ. For example, the NZ maternity care system had established Lead Maternity Carer (LMC) the role of which is to conduct home visits, provide continuity of care during and after pregnancy and educating the mothers and families [30]. It is of paramount importance that these multiple organizations need to work hand-in-hand (adopt intersectoral approach) both among themselves and with the mothers and families especially with Maori and Pacific communities. This will not only promote coordination, communication and collaboration but will also result in the maximum utilization of available services and minimize any duplication. Furthermore, there are lactation support groups within district health boards (DHB's) for providing free advise and support and people should be made aware of it so as to make maximum benefit out of them [32].

Next, for imparting best quality education, the training of health professionals such as nurses, midwives and general practitioners is the next main key area that can hugely affect breast feeding rates. They should be trained in such a way that they are able to provide clear, effective and clinical or evidence based information [29]. Last but not the least, research is one of the crucial component in addressing the effectiveness of any intervention. Thus, breastfeeding statistics including rates (initiation, duration, intensity, ethnicity), health practices in the hospitals and communities, policies and frameworks, should be collected accurately and timely as this will help to make comparisons between the past and present and also to set future targets. Data regarding various enabling factors and barriers (like smoking, plunked visits, cultural beliefs, clinical-poor antenatal education, breast surgery and breast diseases and societal barriers-returning to work early, low socio economic status, unawareness, poor family support) of breastfeeding should also be collected and the above mentioned interventions to promote the enabling factors and minimizing the barriers ultimately will result in good breastfeeding outcomes [33]. On the whole, societal encouragement and acceptance of breastfeeding along with mass media and policy frameworks together will have a huge impact on breastfeeding rates.

\section{Conclusion}

In a conclusion, it can be concluded that NZ has high breastfeeding rates at the time of birth but these slowly decline till 3 and 6 months of age thereby arising the need to address it as one of the public health agenda. The $\mathrm{MOH}$ play a leading role for the protection, promotion and supporting breastfeeding in NZ. There are a number of interventions that had been taken at national, regional and local levels. These measures mainly focus at the initiation but less has been done to continue and support breastfeeding in NZ. Hence, there is a strong need to develop strategies such as establishing a national inter-sectoral breastfeeding committee, implementing Baby Friendly Hospitals throughout New Zealand, gaining active participation of Maori and Pacific whanau, establishing consistent breastfeeding reporting and statistics, increasing breastfeeding promotion, advocacy and coordination, ensuring that pregnant women can access prenatal education and ensuring high quality and ongoing postpartum care for improving the initiation, promotion and maintenance of breastfeeding in New Zealand [8]. All of the above mention implications will contribute towards both maintaining and promoting breastfeeding at 6 months or beyond taking into consideration various barriers and enabling factors.

\section{References}

[1] Marriot, M.B., Campbell, L., Hirsch, E. and Wilson, D. (2007) Preliminary Data from Demographic and Health Surveys on Infant Feeding in 20 Developing Countries. The Journal of Nutrition, 137, 518-523.

[2] Lowdermilk, L.D., Perry, E.S., Cashion, K. and Alden, R.K. (2012) Maternity and Women’s Health Care. 10th Edition, Elsevier Incorporate, St. Louis.

[3] Kotch, B.J. (2005) Maternal and Child Health: Programs, Problems and Policy in Public Health. 2nd Edition, Jones \& 
Bartlett Publishers, Burlington.

[4] Dykes, F. (2006) Breastfeeding in Hospital: Mothers, Midwives and the Production Line. Routledge, London.

[5] Lonnerdal, B. (2000) Breast Milk: A Truly Functional Food. Nutrition, 16, 509-511. http://dx.doi.org/10.1016/S0899-9007(00)00363-4

[6] Imdad, A., Yakoob, Y.M. and Bhutta, A.Z. (2011) Effect of Breastfeeding Promotion Interventions on Breastfeeding Rates, with Special Focus in Developing Countries. BMC Public Health, 11, 14712458. http://dx.doi.org/10.1186/1471-2458-11-s3-s24

[7] Eglash, A., Montgomeery, A. and Wood, J. (2008) Breastfeeding. DM, 5, 343-411. http://dx.doi.org/10.1016/j.disamonth.2008.03.001

[8] National Breastfeeding Advisory Committee of New Zealand. (2009) National Strategic Plan of Action for Breastfeeding 2008-2012: National Breastfeeding Advisory Committee of New Zealand's Advice to the Director-General of Health. Ministry of Health, Wellington.

[9] Royal New Zealand Plunket Society (2010) Breastfeeding Data: Analysis of 2004-2009 Data. Royal New Zealand Plunket Society, Wellington.

[10] Perkins, S. and Vannais, C. (2004) Breastfeeding for Dummies. Wiley Publishing Incorporation, Hoboken.

[11] Ying, W.S. (2005) Breastfeeding: Laws and Societal Impact. Nova Science Publishers, New York. http://dx.doi.org/10.1097/00000446-200505000-00005

[12] Lawrence, M.R. and Pane, A.C. (2007) Human Breast Milk: Current Concepts of Immunology and Infectious Diseases. Current Problem Pediatric Adolescent Health Care, 37, 7-36. http://dx.doi.org/10.1016/j.cppeds.2006.10.002

[13] Newburg, D. (2009) Neonatal Protection by Innate Immune System of Human Milk Consisting of Milk Oligosacchiarides and Glycans. Journal of Animal Science, 87, 26-34. http://dx.doi.org/10.2527/jas.2008-1347

[14] Ogbuanu, I.U., Karmaus, W., Arshad, S.H., Kurukulaaratchy, R.J. and Ewart, S. (2009) Effect of Breastfeeding Duration on Lung Function at Age 10 Years: A Prospective Birth Cohort Study. Thorax, 64, 62-66. http://dx.doi.org/10.1136/thx.2008.101543

[15] Hanson, L. (2004) Protective Effects of Breastfeeding against Urinary Tract Infection. Acta paediatrica, 93, $154-156$. http://dx.doi.org/10.1111/j.1651-2227.2004.tb00695.x

[16] Trotter, S. (2004) Breastfeeding: The Essential Guide. Circa Print Solutions Limited, Renfrewshire.

[17] McKenna, J.J. and McDade, T. (2005) Why Babies Should Never Sleep Alone: A Review of the Co-Sleeping Controversy in Relation to SIDS, Bed Sharing and Breastfeeding. Paediatric Respiratory Reviews, 6, 134-152. http://dx.doi.org/10.1016/j.prrv.2005.03.006

[18] Johnston, P., Flood, K. and Spinks, K. (2004) The Newborn Child. 9th Edition, Elsevier Science, London.

[19] Lauwers. J. and Swisher, A. (2011) Counseling the Nursing Mother: A Lactation Consultant Guide. 5th Edition, Jones and Bartlett Learning, Burlington.

[20] Galson, K.S. (2008) Mothers and Children Benefits From Breastfeeding. Journal of American Dietetic Association, 108, 1106. http://dx.doi.org/10.1016/j.jada.2008.04.028

[21] La Leche League International (2004) The Womanly Art of Breastfeeding. 7th Edition, La Leche League International Incorporation, New York.

[22] Silvia, D.M., Senarath, U., Gunatilake, M. and Lokuhetty, D. (2010) Prolonged Breastfeeding Reduces Risk of Breast Cancer in Sri Lankan Women: A Case-Control Study. Cancer Epidemiology, 34, 267-273. http://dx.doi.org/10.1016/j.canep.2010.02.012

[23] American Dietetic Association (2009) Position of the American Dietetic Association: Promoting and Supporting Breastfeeding. Journal of the American Dietetic Association, 109, 1926-1942. http://dx.doi.org/10.1016/j.jada.2009.09.018

[24] Hall, A.W. and Hauck, Y. (2005) Getting It Right: Australian Primiparas’ View about Breastfeeding: A Quasi Experimental Study. International Journal of Nursing Studies, 44, 786-795. http://dx.doi.org/10.1016/j.ijnurstu.2006.02.006

[25] Wojnar, D. (2004) Maternal Perceptions of Early Breastfeeding Experiences and Breastfeeding Outcomes at 6 Weeks. Clinical Effectiveness in Nursing, 8, 93-100. http://dx.doi.org/10.1016/j.cein.2004.08.001

[26] The National Health Committee (1999) Review of Maternity Health Services in New Zealand. National Health Committee, Wellington.

[27] Jansen, J., Weerth, D.C. and Walraven, R.M.J. (2008) Breastfeeding and the Mother-Infant Relationship-A Review. Developmental Review, 28, 503-521. http://dx.doi.org/10.1016/j.dr.2008.07.001

[28] Department of Labor (2007) Parental Leave in New Zealand: 2005-2006 Evaluation. Department of Labor, Wellington. 
[29] National Breastfeeding Advisory Committee of New Zealand (2008) Background Report: Protecting, Promoting and Supporting Breastfeeding in New Zealand. Ministry of Health, Wellington.

[30] Ministry of Health (2002) Breastfeeding: A Guide to Action. Ministry of Health, Wellington.

[31] New Zealand Breastfeeding Authority (2007) Baby Friendly Hospital Initiative and Baby Friendly Communities Initiative. New Zealand Breastfeeding Authority, New Zealand.

[32] Ministry of Health. (2008) Food and Nutrition Guidelines for Healthy Infants and Toddlers (Aged 0-2): A Background Paper. Ministry of Health, Wellington.

[33] Butler, S., Williams, M., Tukuitonga, C. and Paterson, J. (2004) Factors Associated with Not Breast Feeding Exclusively among Mothers of a Cohort of Pacific Infants in New Zealand. New Zealand Medical Journal, 117, U908. 